\title{
150 Jahre Lungenheilstätte Görbersdorf (Sokołowsko)
}

\section{Years Lungenheilstätte Görbersdorf (Sokołowsko)}

Autor

Institut

\section{A. Jüttemann}

Martin-Luther-Universität Halle-Wittenberg, Institut für Geschichte und Ethik der Medizin (Direktor: Prof. Dr. Florian Steger)
Bibliografie

DOI http://dx.doi.org/

$10.1055 / \mathrm{s}-0034-1365643$

Online-Publikation: 5.6.2014

Pneumologie 2014; 68: 483-487

(c) Georg Thieme Verlag KG

Stuttgart · New York

ISSN 0934-8387

\section{Korrespondenzadresse \\ Andreas Jüttemann, \\ Dipl.-Psych.}

Martin-Luther-Universität

Halle-Wittenberg

Institut für Geschichte und Ethik der Medizin

Magdeburger Str. 8

06112 Halle (Saale)

andreas.juettemann@medizin.

uni-halle.de
Die Errichtung der ersten Tuberkulose-Heilstätte der Welt, von Dr. Hermann Brehmer in Görbersdorf, Niederschlesien (heute Sokołowsko, Polen), gegründet, jährte sich 2013 zum 150. Mal. Im letzten Frühjahr besuchte der Autor im Rahmen eines von ihm an der Berliner Charité durchgeführten Dissertationsprojekts den Ort Görbersdorf, um zu sehen, wieviel von der Einrichtung und dem einstigen Lungenkurort noch erhalten ist.

Der Ort Görbersdorf befindet sich in einem Seitental des Waldenburger Berglands, ein seichter Höhenrücken der Sudeten, der das Riesengebirge mit dem Eulengebirge verbindet. Bis 1945 lag der Ort im Regierungsbezirk Breslau der preußischen Provinz Schlesien. Seitdem gehört die Gemeinde, die nur wenige Kilometer von der Grenze zu Tschechien entfernt ist, zur polnischen Wojewodschaft Niederschlesien (Dolnośląskie). Das Tal von Görbersdorf finden wir, so berichtet Busch 1875, auf einer „schwindsuchtsfreien Höhe.“ Es „dehnt sich mehr in der Länge als in der Breite aus; doch treten die circa 3000 Fuss hohen Berge, welche das Thal von allen Seiten begrenzen, nicht so nahe zu einander, dass es auf den Besucher ein Gefühl der Beklemmung ausübte." [1]

Der junge Arzt Hermann Brehmer, selbst aus Schlesien stammend, suchte einen Ort, an dem die Tuberkulose vorher nicht in Erscheinung getreten war und der außerdem günstige Kurbedingungen bot. Brehmer vertrat damals noch die Auffassung, ein zu kleines Herz sei für die Entstehung der Tuberkulose verantwortlich. Deshalb sollte der Park der von ihm projektierten Heilanstalt eine „Übung des Herzmuskels durch fortgesetzte mäßige Bewegung im Freien“ ermöglichen [2].

Dazu gehörte auch ein langsames Bergaufgehen der Patienten während der Spaziergänge im Park. Aber es gab auch genügend Bänke zum Ausruhen (gemäß der Berg- und Terrainkur nach Oertel ${ }^{1}$ ). Ein zweiter Schwerpunkt von Brehmers Behandlungskonzept war die „reichliche und rationelle
Ernährung der Kranken“. Die Ernährungstherapie bestand aus häufigen Mahlzeiten, abwechslungsreicher Speisenfolge, mit Kognak zugesetzter Milch zur besseren Verdauung und außerdem aus Gaben von Wein gegen Schweißausbruch. Ein dritter Eckpfeiler des Programms zielte auf eine Art Abhärtung: Schlafen bei offenem Fenster, kalte Abreibungen und Duschen sowie eine sog. hydropathische Behandlung [2].

Die von Brehmer begründete Lungenkurtradition reicht bis in das Jahr 1854 zurück, in diesem Jahr übernahm der Arzt von seiner Schwägerin, die recht erfolglos eine Kaltwasserheilanstalt betrieb, ein kleines Haus und behandelte dort drei bis vier Patienten mit Lungentuberkulose. Drei Jahre später, 1857, erhielt Dr. Brehmer durch Alexander von Humboldt, der zu dieser Zeit Mitglied des preußischen Staatsrats war, die staatliche Konzession für den Betrieb einer Kureinrichtung. Daraufhin plante und realisierte Dr. Brehmer ab 1862 den Bau einer Heilanstalt mit Platz für 40 in Einzelzimmern untergebrachten Kranken (Das sog. „Alte Kurhaus“, polnisch „Zamek“ (Schloss) genannt und heute baufällig). Das Haus wurde 1863, also genau vor 150 Jahren, eröffnet [3-5].

Die Anstalt liegt am Hang des Storchbergs. Das Gelände ermöglichte es, die Einrichtung problemlos zu erweitern. Dem Hauptgebäude wurde 1878 ein zusätzlicher Gebäudetrakt („Neues Kurhaus“) angefügt. Damit verfügte das Doppelkurhaus über 110 Zimmer. Zudem gab es, verteilt im Park, drei Villen: das sogenannte „Weiße Haus“ mit 20 Zimmern, das sogenannte „Neue Haus“ mit 15-18 Zimmern, erbaut 1873, und die „Villa Rosa“, heute Pension Róża, mit 16 Zimmern für leichter Erkrankte), ein Wirtschaftsgebäude mit

\footnotetext{
${ }^{1}$ Der Münchener Arzt M. Oertel beschrieb 1886 sog. Terrainkuren als bestes Mittel zur Kräftigung von HerzKreislauf-System und Muskeln. Dafür gab es je nach Leistungsanforderung vier verschiedene Wanderwege mit unterschiedlichen Steigungen.
} 

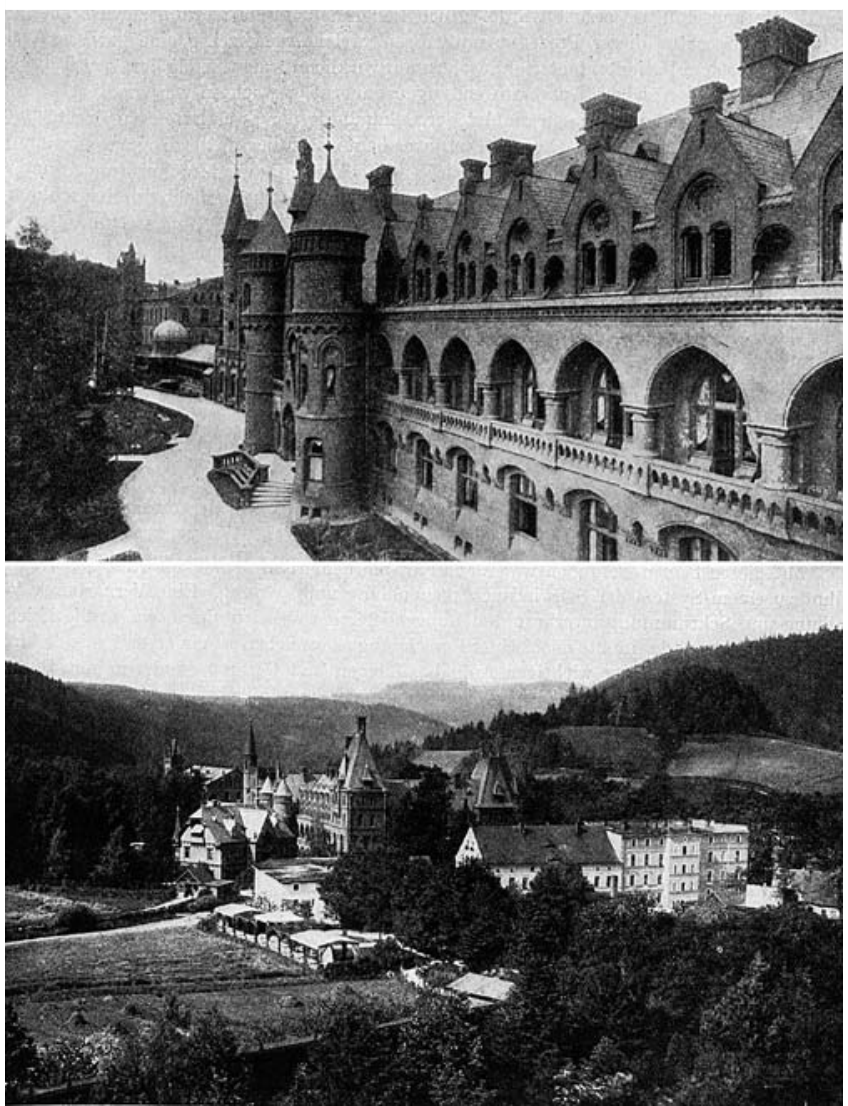

Abb.1 Der erste Heilstättenbau der Welt: Dr. Brehmers „Neues Kurhaus“ im neogotischen Stil, errichtet 1863 in Görbersdorf.

Ställen, die etwas weiter vom Hauptgebäude entfernt lagen, sowie am Südhang des 300 Morgen großen Anstaltsparks ein chemisch-technisches Labor [6].

Das „Weiße Haus“ war der Vorläufer der Anstalt. Dort wurden, wie bereits erwähnt, seit 1854 Lungenkranke behandelt. Der 1863 fertiggestellte Neubau (das „Alte Kurhaus“) war zugleich der Prototyp der ersten eigentlichen Lungenheilstätte der Welt. Alle Neubauten wurden von dem Hannoveraner Architekten und Baurat Oppeler errichtet. Das Kurhaus lässt sich von seinem Stil her der Neogotik zuordnen. Im Jahre 1912 waren alle Gebäude mit Warmwasserheizung und elektrischem Licht ausgestattet. Im „Weißen Haus“ befanden sich später die Wohnung und das Sprechzimmer des 1873 eingestellten Assistenzarztes Peter Dettweiler. Er war übrigens der spätere Begründer der Liegekur und Leiter der Heilstätte Ruppertshain im Taunus [4,7].

Der Nordflügel des „Neuen Kurhauses“ beherbergte im Keller das Inhalatorium für die Behandlung (wie in Bad Ems oder Bad Reichenhall mit Saline) sowie ein Duschbad für die Wintermonate im Sommer wurde im Freien geduscht. Das Alte und das Neue Kurhaus sind durch einen Ergänzungsbau, in dem sich der Wintergarten befand und ein Lesesaal eingerichtet wurde, miteinander verbunden.

Die Villa Rosa und das Neue Haus konnten durch finanzielle Beteiligung von Patienten errichtet werden, die mehrere Jahre mit ihrer Familie in Görbersdorf lebten, um sich von Dr. Brehmer behandeln zu lassen. In den 1920er Jahren kam mit dem „KaiserLogierhaus“ ein weiteres Gebäude für 61 Patienten hinzu. Außerdem gab es eine „Volksheilstätten-Abteilung“ für 100 finanziell minderbemittelte Kranke, die vom Hauptgelände getrennt, in privaten Häusern, verstreut über das Dorf, untergebracht waren. In den Jahren 1925 bis 1929 erfolgte ein Umbau der Heilstätte, um auch die damals neuen chirurgischen Behandlungen durchführen zu können. Ein Operationssaal für aseptische LungenOPs, ein Inhalatorium und ein Strahlentherapieraum wurden eingerichtet. Für die Diagnostik standen ein Röntgengerät (Siemens-Transverter) und ein chemisch-bakteriologisches Labor zur Verfügung. Auch die Liegekur war mittlerweile Bestandteil der Therapie, sodass Waldliegehallen errichtet wurden [7].

Dr. Brehmer wurde in seinem Vorhaben lange Zeit von der Fachwelt belächelt. Er stellte 1855 in einer Studie über die „physiologische Wirkung des veränderten Luftdrucks“ die Behauptung auf, der niedrigere Luftdruck in den Gebirgen übe auf die Tuberkulose eine heilende Wirkung aus. Diese Annahme schien 1856 durch die Untersuchungen von Mühry bestätigt zu werden, der ermittelte, dass Bewohner der Hochgebirge nicht an Tuberkulose litten, wurde aber alsbald verworfen [1].

Obwohl Brehmer erste Heilerfolge in Görbersdorf aufweisen konnte, ignorierten die meisten Kollegen seine Arbeit.

„Diejenigen, welche Notiz von ihm nahmen, unterstützten ihn aber nicht in seinen Bestrebungen, sondern theils verlachten und verspotteten sie ihn, theils (...) stempelten [sie] seine Versuche, die Schwindsucht zu heilen, mit dem Stempel der Charlatanerie; ja sehr fehlte [es] sogar nicht an Leuten, die ihm feindlich entgegentraten und es ihm in jeder Weise erschwerten, eine Heilanstalt, ein Asyl für Lungenkranke errichten zu können.“ [1] Brehmer hatte in Berlin einen großen Befürworter gefunden: Alexander von Humboldt. Humboldt war von den Ideen Brehmers so angetan, dass er auch den prominenten Arzt Schönlein für die Idee begeisterte, in Görbersdorf eine Heilanstalt zu bauen. Er erteilte Brehmer dafür gegen den Widerstand der Fachwelt eine Konzession.

Zunächst kamen nur wenige Tuberkulöse nach Görbersdorf. Doch der Hannoveraner Arzt Dr. Flügge begann nach 1859, Lungenkranke regelmäßig zur Kur in die Brehmer'sche Einrichtung $\mathrm{zu}$ entsenden. Als die ersten Behandelten geheilt (zumindest aber in deutlich besserem Zustand) nach Hannover zurückkehrten, wurden andere Ärzte auf die Heilstätte aufmerksam. Stetig stieg von nun an die Zahl der Kurgäste [1].

Der Ablauf der Kur gestaltete sich wie folgt: Nach zwei Tagen des Aufenthalts, an denen sich die Ankommenden von den Strapazen der Reise erholen, erfolgt die erste Untersuchung durch Dr. Brehmer. Die zweite Untersuchung wird nach einer Woche durchgeführt. Dafür standen drei Assistenzärzte (Dr. Dettweiler, Dr. Greveler und Dr. Sokolowski) zur Verfügung. Nach dem aus Polen stammenden Assistenzarzt Sokolowski erhielt übrigens 1945 Görbersdorf seinen polnischen Namen [4-5].

Im Anschluss an die Untersuchungen bekommen die Patienten Verhaltensanweisungen, die zum einen die Länge und den Ort des Aufenthalts im Freien (eher sonnige oder schattige Plätze) und zum anderen die sportlichen Leistungen (Länge der Spaziergänge und Steigungsgrad der Wege) betreffen [1].

Die sportliche Seite der Kuranwendung nennt Brehmer "methodisches Bergsteigen“. Es soll versucht werden, „die lahmen Lungen, namentlich die oberen Theile" zu aktivieren. Dabei wird das „ärztlich verordnete, systematische Tiefathmen“ praktiziert. Busch bezeichnet dieses Vorgehen als „Gymnastik der kranken Brust“". Das Bergsteigen soll in langsamer Geschwindigkeit erfolgen, damit nicht zu früh eine Ermüdung eintritt und sich die Herzschlagfrequenz und die Atemfrequenz nicht allzu stark erhöhen. Für diese therapeutischen Spaziergänge wird der Anstaltspark genutzt, der sich in Hanglage bis zu einer Höhe von 


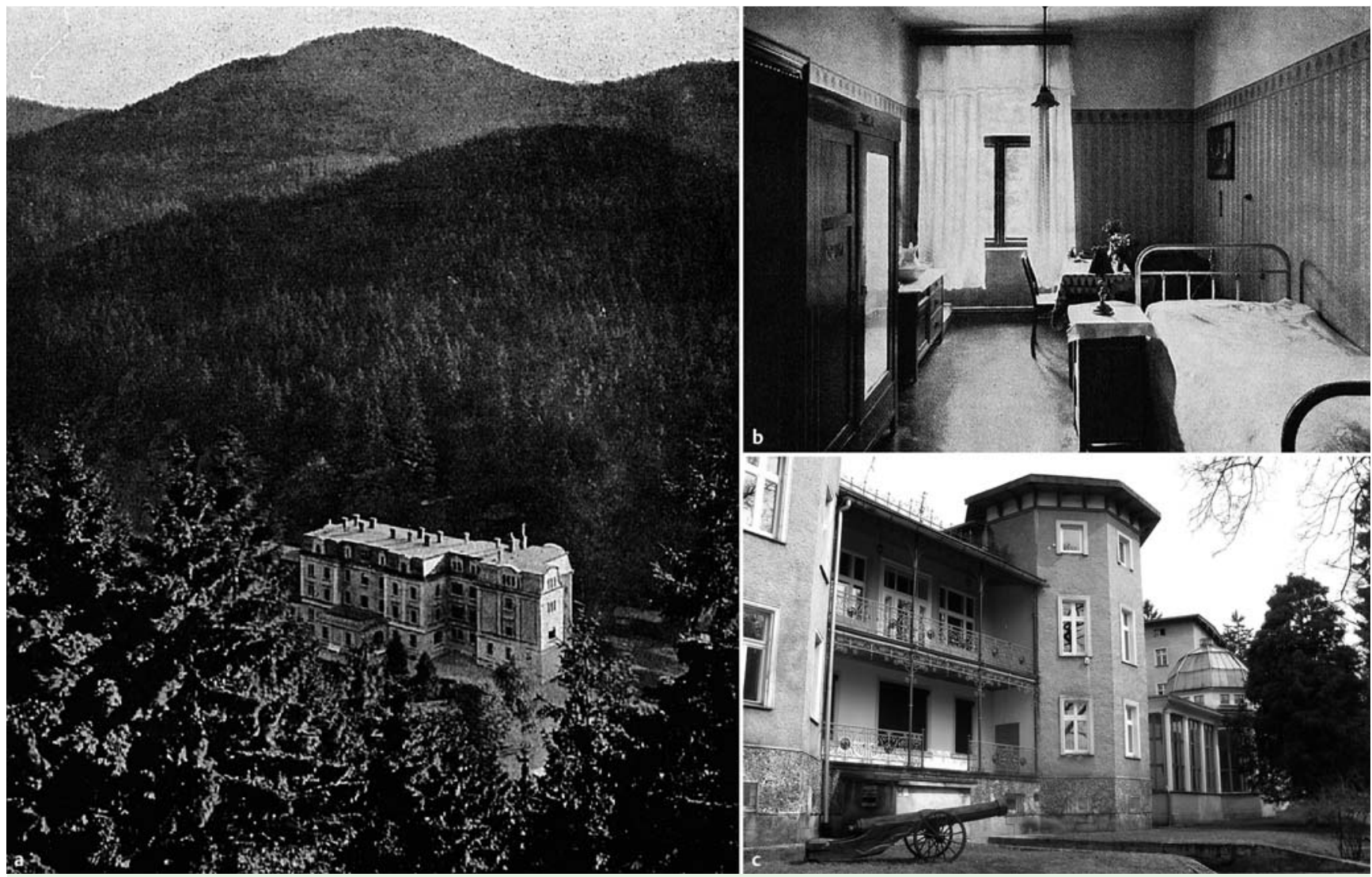

Abb.2 a Konkurrenz für Dr. Brehmer: das Sanatorium des Dr. Weicker am Ortsrand von Görbersdorf, eröffnet 1883. b Konkurrenz für Dr. Brehmer in Görbersdorf: Innenansicht eines Patientenzimmers in Dr. Römplers Heilanstalt, eröffnet 1871 - 75. c Die ehem. Römplersche Anstalt, heute Lungensanatorium Biały Orzeł, 2013.

etwa 790 Metern erstreckt. In Entfernungen von mindestens 50 Schritten sind Ruhebänke angelegt worden [1].

Der Kurtag ist mit fünf Mahlzeiten zeitlich streng gegliedert. Mit dieser Tageseinteilung legt Dr. Brehmer den Grundstein für die später in allen deutschen Lungenheilstätten geltende Diät. Das Wort „Diät“ kennzeichnet eine besonders fett- und nährstoffreiche Ernährung, die eine Gewichtszunahme herbeiführen soll, ganz im Gegensatz zu der heutigen Bedeutung des Begriffs, die in der Regel auf eine Gewichtsreduktion zielt.

Die Verordnung kalorienreicher Nahrung ist in Anlehnung an die damals gültigen wissenschaftlichen Erkenntnisse (genannt wird Dr. Küster in der Deutschen Zeitschrift für praktische Medizin im Jahre 1874) konzipiert worden. Tuberkulöse sollen durch Fette und Kohlehydrate körperlich gestärkt werden. Brehmer rät aber stets zu einer ausgewogenen Ernährung, die auch aus Fleisch und Gemüse besteht, und zwar im Verhältnis 1:4. Bestimmten Patienten wird als Nahrungsergänzung Wein aus Ungarn verabreicht, dessen Anteil an phosporhaltigem Calcium und Magnesium vergleichsweise hoch ist. Etwa 20\% der Kurgäste erhalten einen besonderen Speiseplan, in dem neben ärztlichen Anordnungen auch persönliche Wünsche berücksichtigt worden sind. Die Patienten werden durch Trompetentöne zum Essen gerufen. Das erste Trompetensignal erfolgt bereits um 6:45 Uhr [1]. Einen weiteren Therapiebestandteil bilden kalte Bäder mit einer Wassertemperatur von $6-8^{\circ} \mathrm{C}$. Es handelt sich um sog. „Regenbäder“ (eine Art kalte Dusche). Im Sommer ist für diese Anwendungen ein eigens dafür erbauter Duschpavillon vorgesehen. Im Winter wird die Therapie in einem beheizten Raum im Kurhaus angeboten.
„Diese Bäder, welche vorzugsweise zur Anregung der Hautthätigkeit und zugleich zur Abhärtung des verweichlichten Körpers sowie zur Resorption der betreffenden Exsudate dienen, ihn widerstandsfähiger gegen Witterungseinflüsse zu machen, wurden zuerst von Herrn Dr. Brehmer bei Lungenkranken angewendet, und sollen in gar vielen Fällen wirklich Wunderdinge durch sie bewirkt sein.“ [1]

Direkt im Anschluss an die fünf bis 45 Sekunden lang dauernden Regen- bzw. Duschbäder wird die Haut von einem Bademeister „Warmgerieben“.

Die Ärzte verbringen den ganzen Tag auf dem Gelände des Kurhauses und können bei Fragen oder Problemen jederzeit angesprochen werden. Zudem gibt es zweimal täglich feste Sprechstundentermine des Chefarzts Dr. Brehmer und einmal pro Tag eine Sprechstunde der Assistenzärzte. Für die polnischen Patienten war Dr. Sokolowski zuständig [1].

Das Kurprogramm in Dr. Brehmers Heilanstalt besteht insgesamt aus den folgenden methodischen Komponenten:

- „Ernährungsumstellung (,zweckmäßige Nahrung،)

- Erziehung zu richtiger Ernährung und Lebensweise

- zusätzliche Belebung der Hauttätigkeit zur Unterstützung der schwachen Lunge

- Dämpfung der Blutzirkulation durch den dauerhaft verminderten Luftdruck im Gebirge

- Aufenthalt an der frischen Gebirgsluft

- Bewegung an der frischen Luft im Wechsel mit Ruhe

- Regenbäder, Strahlendusche und kalte Abreibungen

- ständige Kontrolle durch die Ärzte und Pflegekräfte, um die Einhaltung der Kurmethoden sicherzustellen 

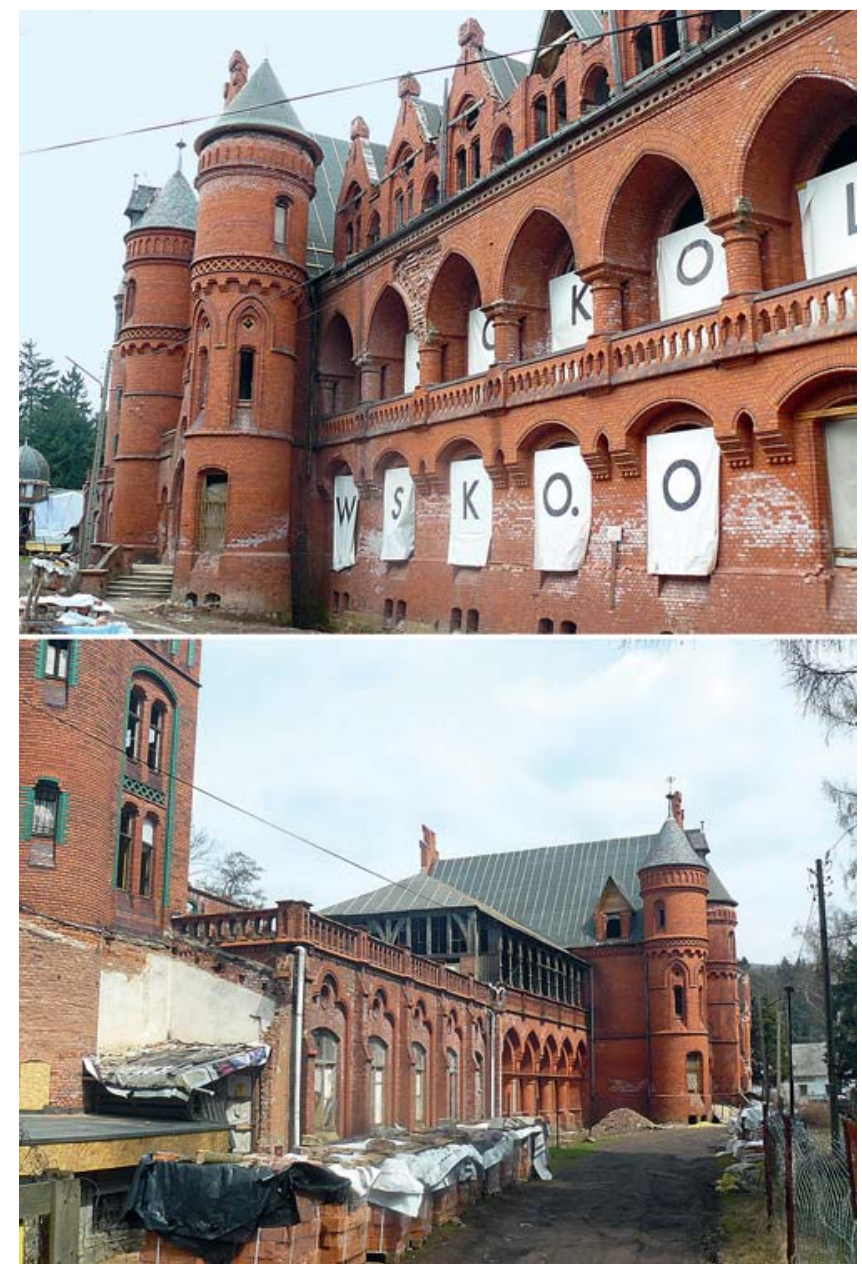

Abb.3 Das baufällige Doppelkurhaus des Dr. Brehmer in Görbersdorf, 2013.

in seltenen Fällen Ergänzung durch medikamentöse Therapie mittels Morphium, Chloral, Pepsin; bei Diarrhöe Rotwein, Pulvis Doweri, Opium und Tannin, bei Affektionen des Larynx Morphium, Priesnitzsche Umschläge, Crotonöl und Inhalation von warmen Dampf. Gegen Blutungen Ergotin innerlich und subcutan in starker Dosis, Eispillen, Eisbeutel und Morphium; außerdem Verdampfungen von Ol. Pul. Pumil. im Schlafzimmer, Carbolsäure innerlich und Ozonwasser“" [1].

Im Kurhaus gibt es einen Wintergarten für Wandelgänge bei schlechtem Wetter. Neben dem Wintergarten befinden sich der „Neue Saal“, in dem man sich zum Lesen (und im Sommer auch zum Speisen) trifft. Hier liegen stets mehrere deutschsprachige sowie einige polnischsprachige Zeitungen und Bücher aus. ${ }^{2}$

Oberhalb der Villa Rosa erhob sich ein „Humboldttempel“ genanntes Gebäude, das 1869 zu Ehren von Alexander von Humboldt und als Dank für seine Verdienste um den Aufbau der Anstalt errichtet wurde. Der Tempel mit der Büste Humboldts existiert heute nicht mehr. Seit 2012 steht auf den Fundamenten des

\footnotetext{
2 Im Jahre 1875 lagen folgende Zeitschriften aus: Kölnische Allgemeine, Augsburger Allgemeine, FAZ, Norddeutsche Allgemeine, Kreuz-Zeitung, Schlesische Presse, Kladderadatsch, die Gartenlaube, Über Land und Meer, Tribüne, Hamburger Reform und die polnische „Czas“. In der 1873 gegründeten Hausbibliothek fanden sich vor allem Werke von Goethe, Schiller, Lessing, Heine, Fritz Reuter, Walter Scott sowie Becker's Weltgeschichte. Außerdem konnten Werke aus der Breslauer und der Waldenburger Stadtbibliothek entliehen werden.
}

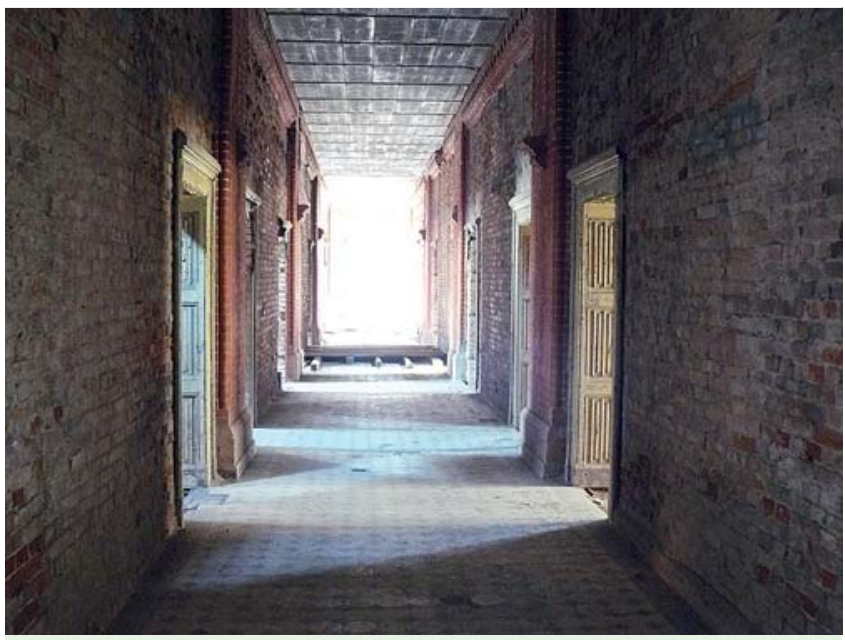

Abb.4 Das Licht fällt schon in die Flure des teilweise dach- und fensterlosen „Neuen Kurhauses“, 2013.

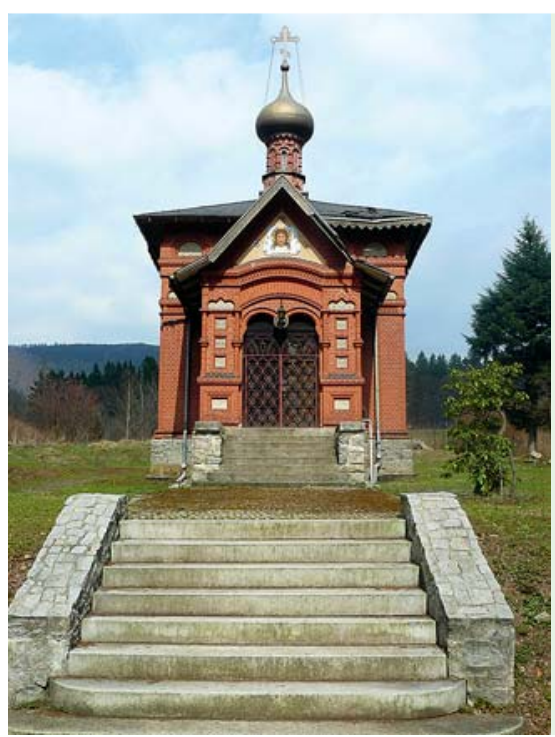

Abb.5 Die orthodoxe Kapelle für die russischen Kurgäste des Sanatoriums Dr. Römpler in Görbersdorf, 2013.

Humboldttempels das Kunstwerk „Łódź postępu“ in Form eines Schiffes des polnischen Künstlers Zbigniew Warpechowski. Zu Brehmers Zeiten wurde im Sommer jeweils 14-tägig ein Konzert im Anstaltspark angeboten, das unweit des Tempels stattfand [3]. Nach Brehmers Tod 1889 war die Anstalt kurz ohne Leitung, da der Fortbestand der Heilstätten nach der Einführung des Tuberkulins ohnehin fraglich war. Doch 1890 machte man weiter. Erster Chefarzt nach Brehmer wurde Felix Wolff. Er verließ bereits 1891 Görbersdorf, und von da an sollte die Klinikleitung öfter wechseln [3].

In den 1950er Jahren wurde ein Teil des Kurhauses abgerissen und das Haus durch den neuen polnischen Chefarzt Dr. Stanislaw Domina von einem Tuberkulosekrankenhaus in eine Fachklinik für Atemwege mit dem Namen „Grunwald“ (der polnische Name der historischen Tannenbergschlacht von 1410) umgewandelt. Im Dezember 1966 wurde die Anstalt geschlossen, nachdem sich die Ortsverwaltung dafür entschieden hatte, die Region als Wintersportgebiet auszubauen [8].

Im Dorf existiert heute noch das Lungenfachkrankenhaus Biały Orzeł, das im fast vollständig erhaltenen ehemaligen Lungensanatorium Dr. Römpler untergebracht ist. Die Einrichtung bestand ab 1874 unweit der Heilstätte von Dr. Brehmer. Die über 


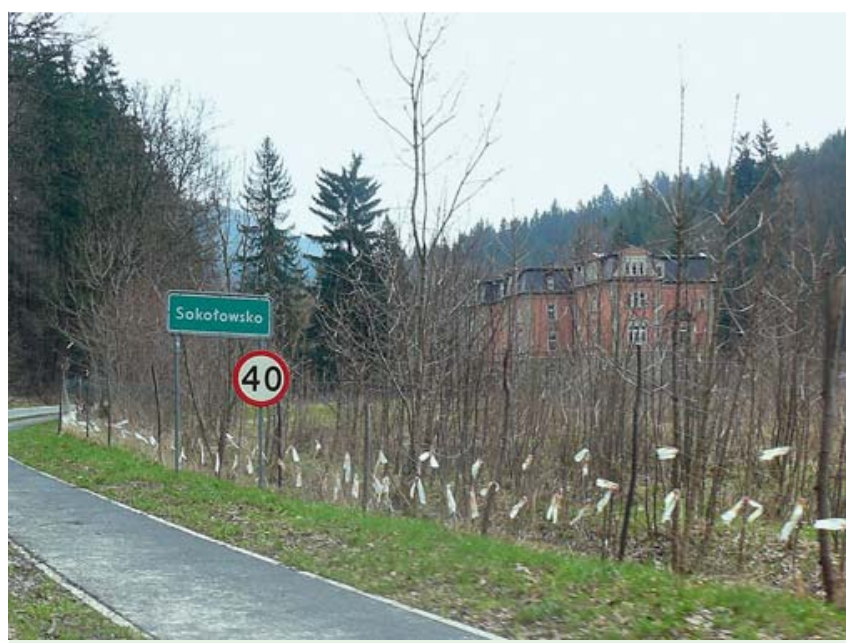

Abb. 6 Ortseingang von Görbersdorf, im Hintergrund ist das Hauptgebäude des ehem. Sanatoriums Dr. Weicker zu sehen, 2013.

das ganze Dorf verstreuten Häuser der einstigen dritten Görbersdorfer Lungenheilanstalt des Dr. Weicker (als „Volksheilstätte“ für Tuberkulosepatienten aus dem Arbeiterstand konzipiert) sind größtenteils erhalten geblieben. Sie wurden zu privaten Wohnhäusern umgebaut [3].

Im Jahre 2005 zerstörte ein Brand weite Teile der noch bestehenden, aber bereits durch Verfall und Vandalismus in Mitleidenschaft gezogenen beiden Hauptgebäude der ehemaligen Brehmer'schen Heilstätte. Der Haupttrakt ist heute in einem besonders ruinösen Zustand. Die Villa Rosa wurde allerdings kürzlich restauriert. Das Weiße und das Neue Haus sind bewohnt, aber sanierungsbedürftig. Derzeit versucht die Warschauer Kulturstiftung „In Situ“, die 2008 das gesamte sechs Hektar große Anstaltsgelände erworben hat, das Neue Kurhaus wieder aufzubauen und für eine kulturelle Nutzung verfügbar zu machen. Auf dem Gelände des in den 1950er Jahren abgetragenen Gebäudeteils des Kurhauses ist die Errichtung eines Neubaus geplant. Wenngleich der laufende Wiederaufbau wegen der spärlich fließenen Spendengelder nur langsam voranschreitet, werden auf dem Gelände von der Stiftung bereits Kulturprojekte (Filmfestivals, Workshops und Ausstellung) durchgeführt [8].

\section{Link}

Internetseiten zum Dissertationsprojekt des Autors und zu den Heilstätten im Riesengebirge (mit weiteren Bildern seines Besuchs): www.lungenheilstaetten.de und www.goerbersdorf.de

\section{Literatur}

1 Busch HK. Die Görbersdorfer Heilanstalt des Dr. H. Brehmer. Eine Klinik für chronisch Lungenkranke Berlin: Enslin; 1875

2 von Leyden E. Fest-Rede zur Feier des fünfzigjährigen Bestehens von Dr. Brehmers Heilanstalten für Lungenkranke in Görbersdorf i. Schlesien. Wiesbaden: Bergmann; 1904

3 Averbeck $H$. Von der Kaltwasserkur bis zur physikalischen Therapie: Betrachtungen zu Personen und zur Zeit der wichtigsten Entwicklungen im 19. Jahrhundert. Norderstedt: BoD; 2012

4 Hähner-Rombach S. Sozialgeschichte der Tuberkulose. Stuttgart: Steiner; 2000

5 Langerbeins I. Lungenheilanstalten in Deutschland. Köln: Univ. Diss; 1979

6 Welten 0 . Die Heilanstalten von Görbersdorf. Was sie versprechen und was sie halten Für Aerzte und Kranke auf Grund eigener Anschauung und authentischer Quellen dargestellt von Dr. Oskar Welten. Berlin: Issleib; 1888

7 Helm F. Tuberkulose-Heilstätten im Deutschen Reich. Düsseldorf: Rhenania; 1930

8 Internetseite der Kulturstiftung InSitu zur Geschichte Görbersdorfs Abgerufen unter sokolowsko.org/soko\%C5\%82owsko/historia am 21. November 2013 